\title{
I.APRESENTAÇÃO [p.19]
}

\section{II.INTRODUÇÃO [p.25]}

III.BIBLIOGRAFIA E INSTRUMENTOS DE TRABALHO [p.43]

\section{IV.INVENTÁRIO DOS FUNDOS MONÁSTICO-CONVENTUAIS DO DISTRITO DE ÉVORA [p.67]}

\section{A - ORDENS [p.69]}

A.I. CÓNEGOS REGULARES [p.69]

CONGREGAÇÃO DOS CÓNEGOS SECULARES DE SÃO JOÃO EVANGELISTA (GF) [p.69]

Conventos da Congregação dos Cónegos Seculares de São João Evangelista (SGF) [p.70]

Convento de São João Evangelista de Évora (F) [p.70]

Hospital de Portel (SF) [p.73]

Convento de Nossa Senhora da Assunção de Arraiolos (F) [p.73]

A.II. ORDENS MONÁSTICAS [p.77]

ORDEM DA CARTUXA (GF) [p.77]

Mosteiro da Scala Coeli de Évora (F) [p.77]

ORDEM DE CISTER (GF) [p.81]

Mosteiros da Congregação de Santa Maria de Alcobaça (SGF) [p.82]

Mosteiros Femininos [p.82]

Mosteiro de São Bento de Cástris (F) [p.82]

ORDEM DE SÃO PAULO PRIMEIRO EREMITA DA CONGREGAÇÃO DA SERRA DE OSSA (GF) [p.95]

Mosteiros da Congregação de São Paulo da Serra de Ossa (SGF) [p.96]

Província da Congregação de São Paulo da Serra de Ossa (F) [p.96]

Mosteiro de São Paulo da Serra de Ossa (F) [p.100]

Mosteiro de Santo Onofre de Vale Abraão (SF) [p.106]

Mosteiro de Santo Antão de Vale de Infante (SF) [p.106]

Mosteiro de Nossa Senhora do Amparo de Vila Viçosa (F) [p.107]

Mosteiro de São Paulo ou de Nossa Senhora do Socorro de Portel (F) [p.109]

Mosteiro de São Paulo de Borba (F) [p.111]

Colégio de Nossa Senhora da Soledade de Borba (SF) [p. 112] 
Colégio de São Paulo de Évora (F) [p.114]

Mosteiro de Santa Catarina de Montemuro [p.116]

ORDEM DE SÃO JERÓNIMO (GF) [p.117]

Mosteiros da Província de Portugal (SGF) [p.117]

Mosteiros Masculinos [p.118]

Mosteiros de Nossa Senhora do Espinheiro de Évora (F) [p.118]

Mosteiros Femininos [p.126]

Mosteiro do Bom Jesus de Viana do Alentejo (F) [p.126]

A.III.ORDENS MENDICANTES [p.133]

ORDEM DOS FRADES MENORES (GF)[p.133]

Conventos da Província da Piedade (SGF) [p.136]

Província da Piedade (F) [p.137]

Convento de Nossa Senhora da Consolação do Bosque de Borba (F) [p.137]

Convento de Santo António de Estremoz (F) [p.39]

Convento do Bom Jesus de Valverde de Évora (F) [p.139]

Convento de Santo António de Évora (F) [p.140]

Convento de Santo António do Redondo (F) [p.140]

Conventos da Província dos Algarves (SGF) [p.141]

Conventos Masculinos [p. 141]

Convento de São Francisco de Évora (F) [p.141]

Convento de São Francisco de Estremoz (F) [p.145]

Convento de São Francisco de Montemor-o-Novo (F) [p.148]

Mosteiros Femininos [p.149]

Mosteiro de Santa Clara de Évora (F) [p.149]

Mosteiro das Chagas de Vila Viçosa (F) [p.157]

Mosteiro de Nossa Senhora da Esperança de Vila Viçosa (F) [p.162]

Mosteiro do Calvário de Évora (F) [p.169]

Mosteiro das Servas de Nossa Senhora de Borba (F) [p.171] 
Conventos da Província da Terceira Ordem da Penitência (SGF) [p.175]

Conventos Masculinos [p.176]

Convento de São Francisco de Viana do Alentejo (F) [p.176]

Colégio do Espírito Santo de Évora (F) [p.177]

Convento de São Francisco do Vimieiro (F) [p.181]

Convento de São Francisco de Arraiolos (F) [p.181]

Mosteiros de Jurisdição Diocesana (SGF) [p.183]

Mosteiro do Salvador de Évora (F) [p.183]

Mosteiro do Santíssimo Sacramento e Luz de Montemor-o-Novo (F) [p.196]

Irmandade de Nossa Senhora da Luz (SF) [p.197]

ORDEM DOS PEGADORES (GF) [p.198]

Conventos da Província de Portugal (SGF) [p.199]

Conventos Masculinos [p.200]

Convento de São Domingos de Évora (F) [p.200]

Confraria de Nossa Senhora do Rosário (SF) [p.202]

Convento de Santo António de Lisboa de Montemor-o-Novo (F) [p.202]

Mosteiros Femininos [p.204]

Mosteiro de Santa Catarina de Sena de Évora (F) [p.204]

Mosteiro de Nossa Senhora do Paraíso de Évora (F) [p.210]

Mosteiro de Nossa Senhora da Saudação de Montemor-o-Novo (F) [p.220]

ORDEM DO CARMO (GF) [p.223]

Conventos da Província de Portugal (SGF) [p.224]

Conventos Masculinos [p.225]

Convento de Nossa Senhora do Carmo ou da Luz de Évora (F) [p.225]

Ordem Terceira do Carmo (SGF) [p.227]

Ordem Terceira de Nossa Senhora do Carmo de Portel (F) [p.227]

ORDEM DOS CARMELITAS DESCALÇOS (GF) [p.227]

Conventos da Província de São Filipe de Portugal (SGF) [p.228] 
Conventos Masculinos [p.228]

Convento de Nossa Senhora dos Remédios de Évora (F) [p.228]

Mosteiros Femininos [p.230]

Mosteiro de São José de Évora (F) [p.230]

Recolhimentos (SGF) [p.233]

Recolhimentos de Santa Marta de Évora (F) [p.234]

ORDEM DOS EREMITAS DE SANTO AGOSTINHO (GF) [p.235]

Conventos da Província de Portugal (SGF) [p.235]

Conventos Masculinos [p.236]

Convento de Santo Agostinho de Vila Viçosa (F) [p.236]

Convento de Nossa Senhora da Graça de Évora (F) [p.238]

Confraria de Nossa Senhora da Graça (SF) [p.243]

Confraria de Santa Luzia (SF) [p.244]

Mosteiros Femininos [p.244]

Mosteiro de Santa Mónica de Évora (F) [p.244]

Confraria do Senhor Menino Jesus (SF) [p.252]

Mosteiro de Santa Cruz e Luz de Vila Viçosa (F) [p.252]

Confraria do Santíssimo Sacramento (SF) [p.256]

CONGREGAÇÃO DOS EREMITAS DESCALÇOS DE SANTO AGOSTINHO (GF) [p.256]

Conventos da Congregação de Portugal (SGF) [p.257]

Conventos Masculinos [p.257]

Convento de Nossa Senhora das Mercês de Évora (F) [p.257]

Convento de Nossa Senhora da Consolação de Estremoz (F) [p.257]

Convento de Nossa Senhora da Conceição de Montemor-o-Novo (F) [p.259]

Hospício de São Nicolau de Tolentino de Mora (F) [p.260]

ORDEM HOSPITALEIRA DE SÃO JOÃO DE DEUS (GF) [p.260]

Conventos da Província de Portugal (SGF) [p.261]

Convento de São João de Deus de Montemor-o-Novo (F) [p.262] 


\section{A.IV.CLÉRIGOS REGULARES [p.263]}

COMPANHIA DE JESUS (GF) [p.263]

Casas Professas e Colégios da Província de Portugal (CGF) [p.264]

Colégio da Madre de Deus de Évora (F) [p.265]

CONGREGAÇÃO LUSITANA DOS CLÉRIGOS REGULARES MINISTROS DOS ENFERMOS (GF) [p.266]

Conventos da Congregação Lusitana dos Clérigos Regulares Ministros dos Enfermos (SGF) [p.267]

Convento de Nossa Senhora do Alcance de Mourão (F) [p.267]

\section{A.V.ORDENS MILITARES [p.268]}

ORDEM DO HOSPITAL (GF) [p.268]

Conventos Femininos [p.269]

Convento de São João da Penitência de Estremoz (F) [p.269]

Confraria da Santíssima Trindade (SF) [p.277]

Irmandade de Nossa Senhora da Assunção e Ressurreição de Cristo (SF) [p.277]

Irmandade do Santíssimo Sacramento (SF) [p.279]

CONGREGAÇÃO DO ORATÓRIO DE SÃO FILIPE DE NÉRI (GF) [p.279]

Casas da Congregação em Portugal (SGF) [p.280]

Congregação do Oratório de Nossa Senhora da Conceição de Estremoz (F) [p.280]

CONGREGAÇÃO DA MISSÃO (GF) [p.284]

Colégio de Nossa Senhora da Purificação de Évora [p.285]

V.OUTRA DOCUMENTAÇÃO MONÁSTICO-CONVENTUAL INCORPORADA NA BIBLIOTECA PÚBLICA DE ÉVORA [p.287]
A - ORDENS [p.289]

\section{A.I.CONÉGOS REGULARES [p.289]}

ORDEM DOS CÓNEGOS REGULARES DE SANTO AGOSTINHO [p.289]

Congregação de Santa Cruz de Coimbra [p.289]

Conventos Masculinos [p.289]

Convento de Santa Cruz de Coimbra [p.289]

Convento de São Vicente de Fora (Lisboa) [p.290] 
Convento do Salvador do Banho [p.291]

Mosteiros Femininos [p.291]

Mosteiro de Santa Maria de Chelas [p.291]

CONGREGAÇÃO DOS CÓNEGOS SECULARES DE SÃO JOÃO EVANGELISTA [p.291]

Convento de São Bento de Xabregas (Lisboa) [p.291]

A.II.ORDENS MONÁSTICAS [p.292]

ORDEM DE SÃO BENTO [p.292]

Congregação de São Bento de Portugal [p.292]

ORDEM DE CISTER [p.293]

Congregação de Santa Maria de Alcobaça [p.293]

ORDEM DE SÃO PAULO PRIMEIRO EREMITA DA CONGREGAÇÃO DA SERRA DE OSSA [p.294]

Mosteiro de Nossa Senhora da Rosa da Caparica [p.294]

ORDEM DO SANTÍSSIMO SALVADOR [p.294]

Mosteiro de Nossa Senhora da Conceição de Marvila [p.295]

ORDEM DE SÃO JERÓNIMO [p.295]

Província de Portugal [p.295]

Mosteiros Masculinos [p.296]

Mosteiro de São Marcos de Coimbra [p.296]

Mosteiro de Santa Maria de Belém (Lisboa) [p.296]

A.III.ORDENS MENDICANTES [p.297]

ORDEM DOS FRADES MENORES [p.297]

Convento e Seminário Apostólico de Santo António do Varatojo [p.297]

Hospício da Terra Santa [p.298]

Conventos da Província de Portugal [p.298]

Província de Portugal [p.299]

Conventos Masculinos [p.299]

Convento de Santa Zita da Asseiceira de Tomar (ou São Francisco de Valbom) [p.299]

Mosteiros Femininos [p.300] 
Mosteiros de Santa Clara de Lisboa [p.300]

Conventos da Província da Piedade [p.300]

Convento de Santo António de Beja [p.300]

Conventos da Província dos Algarves [p.301]

Província dos Algarves [p.301]

Conventos Masculinos [p.301]

Convento de São Francisco de Portalegre [p.301]

Convento de São Francisco de Beja [p.302]

Convento de Nossa Senhora da Visitação de Vila Verde dos Francos [p.302]

Mosteiros Femininos [p.302]

Mosteiro de Nossa Senhora da Conceição de Beja [p.302]

Mosteiro da Madre de Deus de Lisboa [p.304]

Mosteiro da Santa Clara de Elvas [p.305]

Mosteiro de Nossa Senhora de Aracoeli de Alcácer do Sal [p.305]

Conventos da Província da Arrábida [p.305]

Província da Arrábida [p.306]

Convento de Santa Maria de Jesus do Vale de Figueira [p.306]

Convento de Nossa Senhora e Santo António de Mafra [p.306]

Conventos da Província de Santo António [p.307]

Província de Santo António [p.307]

Conventos da Província de São Tomé, na Índia Oriental [p.307]

Província de São Tomé [p.308]

Conventos da Província da Madre de Deus, na Índia Oriental [p.308]

Província da Madre de Deus [p.308]

Conventos Masculinos [p.309]

Convento da Madre de Deus de Daugim (Goa) [p.309]

Convento de Nossa Senhora dos Anjos de Diu [p.309]

Hospício da Porciúncula ou de Nossa Senhora dos Anjos de Rachol [p.310] 
Conventos da Província da Soledade [p.311]

Província da Soledade [p.311]

Conventos da Província da Conceição de Portugal [p.311]

Província da Conceição de Portugal [p.311]

Conventos da Província da Terceira Ordem da Penitência [p.312]

Província da Terceira Ordem da Penitência [p.312]

Conventos Masculinos [p.312]

Convento de Santa Catarina de Vale de Mourol de Santarém [p.312]

Convento de Nossa Senhora de Jesus de Lisboa [p.313]

Mosteiros de Jurisdição Diocesana [p.313]

Mosteiro de Santa Clara de Guimarães [p.313]

Mosteiro de Santa Marta de Lisboa [p.314]

Mosteiro de Terceiras Regulares de Jurisdição Diocesana [p.314]

Mosteiro de Nossa Senhora da Graça do Torrão [p.314]

Recolhimentos [p.315]

Recolhimento de Santa Maria dos Inocentes de Santarém [p.315]

ORDEM DA CONCEIÇÃO DE MARIA [p.315]

ORDEM DOS PREGADORES [p.316]

Conventos da Província de Portugal [p.316]

Província de Portugal [p.316]

Conventos Masculinos [p.316]

Convento de Montejunto [p.316]

Convento de São Domingos ou de Nossa Senhora da Oliveira de Santarém [p.317]

Convento de São Domingos de Lisboa [p.317]

Mosteiros Femininos [p.318]

Mosteiro do Salvador de Lisboa [p.318]

Mosteiro de Jesus de Aveiro [p.319]

Mosteiro de Nossa Senhora da Assunção do Castelo de Moura [p.319] 
Conventos da Congregação da Índia Oriental [p.320]

Congregação da Índia Oriental [p.320]

ORDEM DO CARMO [p.320]

Conventos da Província de Portugal [p.320]

Província de Portugal [p.320]

Mosteiros Femininos [p.321]

Mosteiro de Nossa Senhora da Esperança de Beja [p.321]

Recolhimentos [p.321]

Recolhimentos de Nossa Senhora do Carmo de Cuba [p.321]

ORDEM DOS CARMELITAS DESCALÇOS [p.322]

Província de São Filipe de Portugal [p.322]

Conventos Masculinos [p.322]

Convento de Nossa Senhora dos Remédios de Lisboa [p.322]

Convento de São João da Cruz de Carnaxide [p.323]

Mosteiros Femininos [p.323]

Mosteiro e Basílica do Sagrado Coração de Jesus (Estrela, Lisboa) [p.323]

ORDEM DOS EREMITAS DE SANTO AGOSTINHO [p.323]

Conventos da Província de Portugal [p.323]

Província de Portugal [p.323]

Conventos Masculinos [p.324]

Colégio de Nossa Senhora da Graça de Coimbra [p.324]

Convento de Santo Agostinho ou de São João Novo do Porto [p.325]

Convento de Nossa Senhora da Penha de França (Lisboa) [p.325]

Mosteiros Femininos [p.326]

Mosteiro de Santa Ana de Coimbra [p.326]

Conventos da Congregação da Índia Oriental [p.326]

Congregação da Índia Oriental [p.326]

Conventos Masculinos [p.324] 
Convento de Nossa Senhora da Graça de Goa [p.327]

Mosteiros Femininos [p.327]

Mosteiro de Santa Mónica de Goa [p.327]

CONGREGAÇÃO DOS EREMITAS DESCALÇOS DE SANTO AGOSTINHO [p.328]

Congregação de Portugal [p.328]

Mosteiros Femininos [p.328]

Mosteiro de Nossa Senhora da Conceição de Vale de Xabregas [p.328]

ORDEM DOS MÍNIMOS [p.328]

Província de Portugal [p.329]

ORDEM DA SANTÍSSIMA TRINDADE [p.329]

Província de Portugal [p.330]

Convento da Santíssima Trindade de Santarém [p.331]

ORDEM HOSPITALEIRA DE SÃO JOÃO DE DEUS [p.331]

Província de Portugal [p.331]

A.IV.CLÉRIGOS REGULARES [p.331]

COMPANHIA DE JESUS [p.331]

Casas Professas e Colégios da Província de Portugal [p.331]

Província de Portugal [p.331]

Colégio de Jesus de Coimbra [p.333]

Casa Professa de São Roque de Lisboa [p.334]

Colégio de São Sebastião de Portalegre [p.335]

Casas Professas e Colégios da Província de Goa [p.335]

Casa Professa do Bom Jesus de Goa [p.335]

Casas Professas e Colégios da Vice-Província do Maranhão e Grão-Pará (Brasil) [p.336]

Vice-Província do Maranhão e Grão-Pará [p.336]

ORDEM DOS CLÉRIGOS REGULARES [p.337]

Casa de Nossa Senhora da Divina Providência (Lisboa) [p.337]

CONGREGAÇÃO LUSITANA DOS CLÉRIGOS REGULARES MINISTROS DOS ENFERMOS [p.338] 
Convento de Nossa Senhora das Necessidades da Tomina [p.338]

A.V.ORDENS MILITARES [p.338]

ORDEM DO HOSPITAL [p.338]

ORDEM DE SANTIAGO [p.339]

Conventos Femininos [p.340]

Convento de Santos [p.340]

ORDEM DE AVIS [p.340]

Conventos Masculinos [p.341]

Convento de São Bento de Avis [p.341]

Conventos Femininos [p.341]

Convento de Nossa Senhora da Encarnação (Lisboa) [p.341]

ORDEM DE CRISTO [p.342]

Conventos Masculinos [p.343]

Convento de Nossa Senhora da Luz de Carnide [p.343]

Colégio de Nossa Senhora da Conceição de Coimbra [p.343]

B - SOCIEDADES DE VIDA APOSTÓLICA [p.344]

CONGREGAÇÃO DO ORATÓRIO DE SÃO FILIPE DE NÉRI [p.344]

Casas da Congregação em Portugal [p.344]

Congregação Portuguesa [p.344]

Congregação do Oratório de Espírito Santo da Pedreira (Lisboa) [p.344]

Hospício do Oratório de Nossa Senhora das Necessidades de Lisboa [p.345]

Casas da Congregação do Oratório de Goa [p.345]

Congregação do Oratório de Santa Cruz dos Milagres de Goa [p.345]

VI.DOCUMENTAÇÃO MONÁSTICO-CONVENTUAL DE ORIGEM INDETERMINADA [p.347] 